\title{
Installation of Vascular Interventional Lab for Animal Model Studies at PGIMER, Chandigarh: Beginning of a New Era
}

\author{
Ajay Kumar ${ }^{1}$ Manavjit Singh Sandhu ${ }^{1}$ Paramjeet Singh ${ }^{1} \quad$ Naveen Kalra $^{1}$ Indu Verma ${ }^{2}$ Vikas Bhatia ${ }^{1}$
}

${ }^{1}$ Department of Radio-diagnosis and Imaging, Postgraduate Institute of Medical Education and Research (PGIMER), Chandigarh, India

2 Department of Biochemistry, Postgraduate Institute of Medical Education and Research (PGIMER), Chandigarh, India

J Clin Interv Radiol ISVIR 2023;7:69-72.

Address for correspondence Ajay Kumar, MD, Department of RadioDiagnosis and Imaging, Postgraduate Institute of Medical Education and Research (PGIMER), Chandigarh 160012, India (e-mail: ajay2509@gmail.com).

\section{Anesthetic Considerations}

One of the key challenges is to provide adequate anesthesia to animals for completion of the diagnostic or therapeutic research procedure. Many different types of anesthesia protocols have been described previously. ${ }^{1-3}$ With limited experience and technical hardware for animal resuscitation, we had to decide on an anesthesia protocol that was feasible, safe, provides immobilization for 45 to 60 minutes, and the animal can be maintained on room air. New Zealand rabbits were anesthetized intramuscularly in the groin muscles (-Fig. 1) with ketamine $\mathrm{HCl}(50 \mathrm{mg} / \mathrm{kg})$ and xylazine $(5$ $\mathrm{mg} / \mathrm{kg}$ ) and maintained on room air. This provides adequate sedation and immobilization for our procedures.

\section{Animal Positioning}

After sedation, it is important to place the animal in an adequate position for proper exposure of the vascular access site. For this, a custom-made wooden table was used that allowed each of the four extremities to be fixated with straps (-Fig. 1).

It is crucial to select an appropriate animal model that could be easily handled, technically feasible to perform catheterization and a repeat study could be achieved. We selected the New Zealand white rabbit for this purpose. The main reasons were easy availability at our hospital, easy to handle the animal, similar coagulation profile and blood pressure to humans. ${ }^{1}$

In addition, the profile, size and diameter of rabbit neck vessels are similar to human cerebral vasculature. The New Zealand white rabbit is an already established experimental animal for research and innovation in neurovascular interventions. ${ }^{1,2}$

article published online March 4, 2022
DOI https://doi.org/ $10.1055 / \mathrm{s}-0042-1743485$. ISSN 2457-0214.

\section{Vascular Access}

Another key challenge is to get vascular access for selective catherization in New Zealand white rabbits as the vessels are tiny in diameter. We employed transauricular (-Fig. 2) and transfemoral (-Fig. 3) routes for access. The techniques for these have been previously described. ${ }^{2,3}$ Transfemoral route requires a cut down procedure, which is technically challenging during the initial learning phases. A $3 \mathrm{~F}$ sheath or $18 \mathrm{G}$ cannula was used for the initial puncture and arterial access.

(C) 2022. Indian Society of Vascular and Interventional Radiology. All rights reserved.

This is an open access article published by Thieme under the terms of the Creative Commons Attribution-NonDerivative-NonCommercial-License, permitting copying and reproduction so long as the original work is given appropriate credit. Contents may not be used for commercial purposes, or adapted, remixed, transformed or built upon. (https://creativecommons.org/ licenses/by-nc-nd/4.0/)

Thieme Medical and Scientific Publishers Pvt. Ltd., A-12, 2nd Floor, Sector 2, Noida-201301 UP, India 


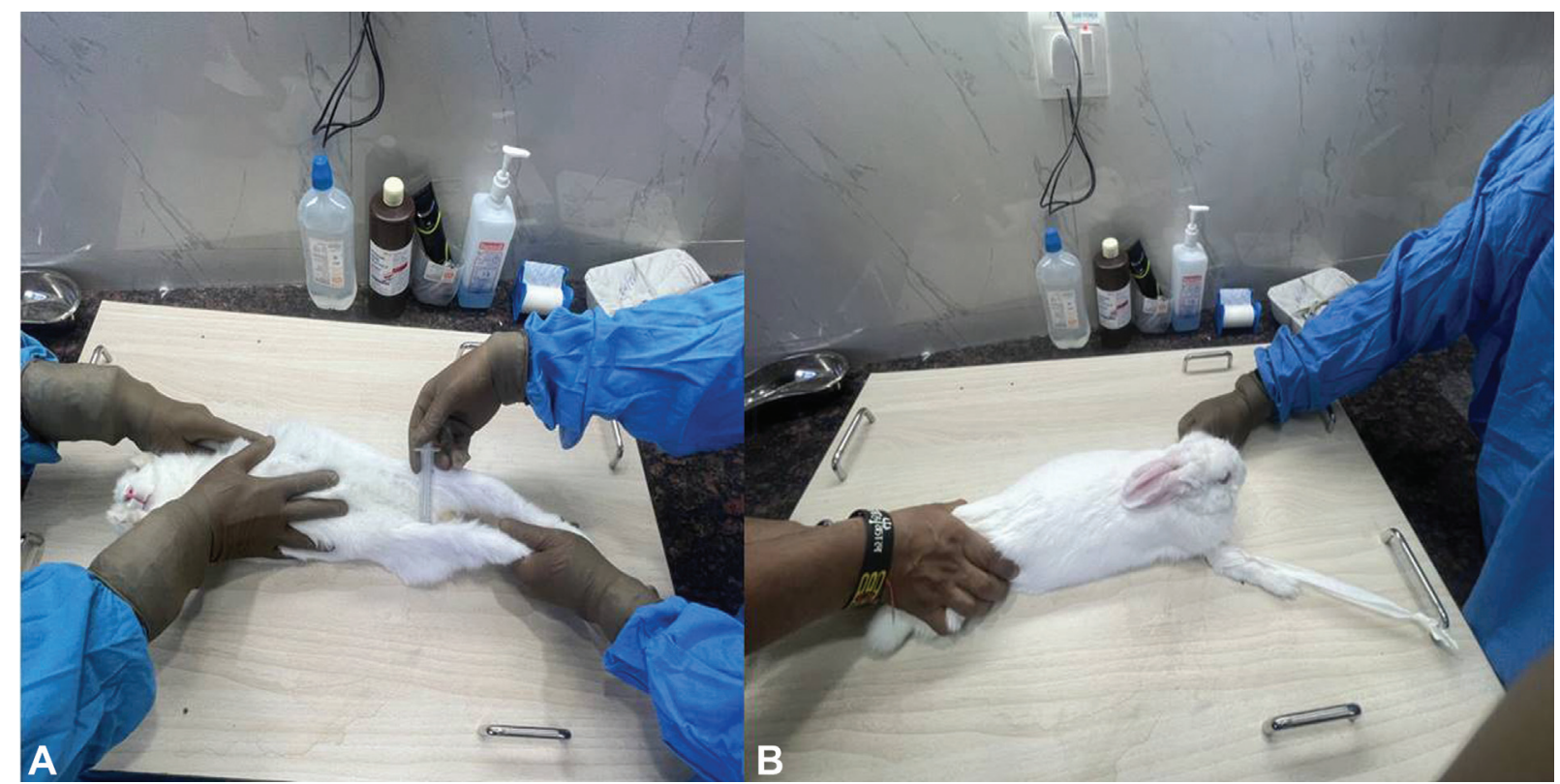

Fig. 1 (A) Intramuscular injection of anesthetic agents to the New Zealand white rabbit. (B) Securing the limbs prior to procedure on the custommade wooden holding table.

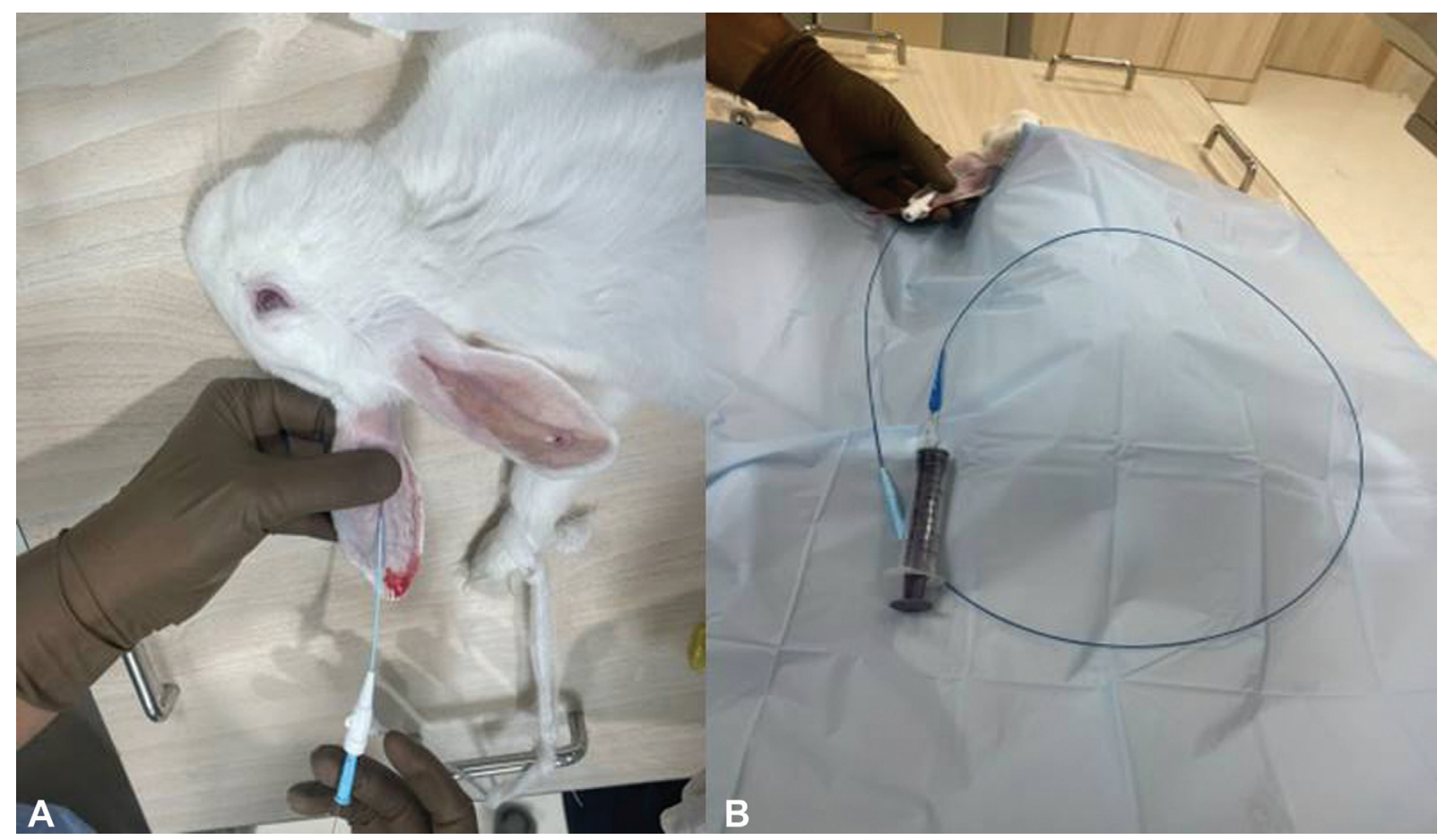

Fig. 2 (A) The presence of a $3 \mathrm{~F}$ sheath in the central auricular artery of the left ear. (B) Placement of a 1.7-F microcatheter through the $3 \mathrm{~F}$ sheath for selective angiography.

\section{Selective Catheterization}

Maneuvering the catheter in small vessels is challenging initially and requires technical expertise. Using 3-F catheters or coaxial systems with $1.7-\mathrm{F}$ or $1.9-\mathrm{F}$ micro catheters, we could easily navigate and perform intracranial angiography with excellent images (- Fig. 4). The technical steps for selective catheterization of intracranial vessels have been previously described. ${ }^{2,3}$

\section{Current Projects}

Multiple projects are currently running from the department of Radio-diagnosis and Imaging that are approved by the institutional animal ethical committee.

These include performing transfemoral angiography, transauricular angiography, to study variations of Circle of Willis in New Zealand white rabbits and creation of aneurysm flow models. 


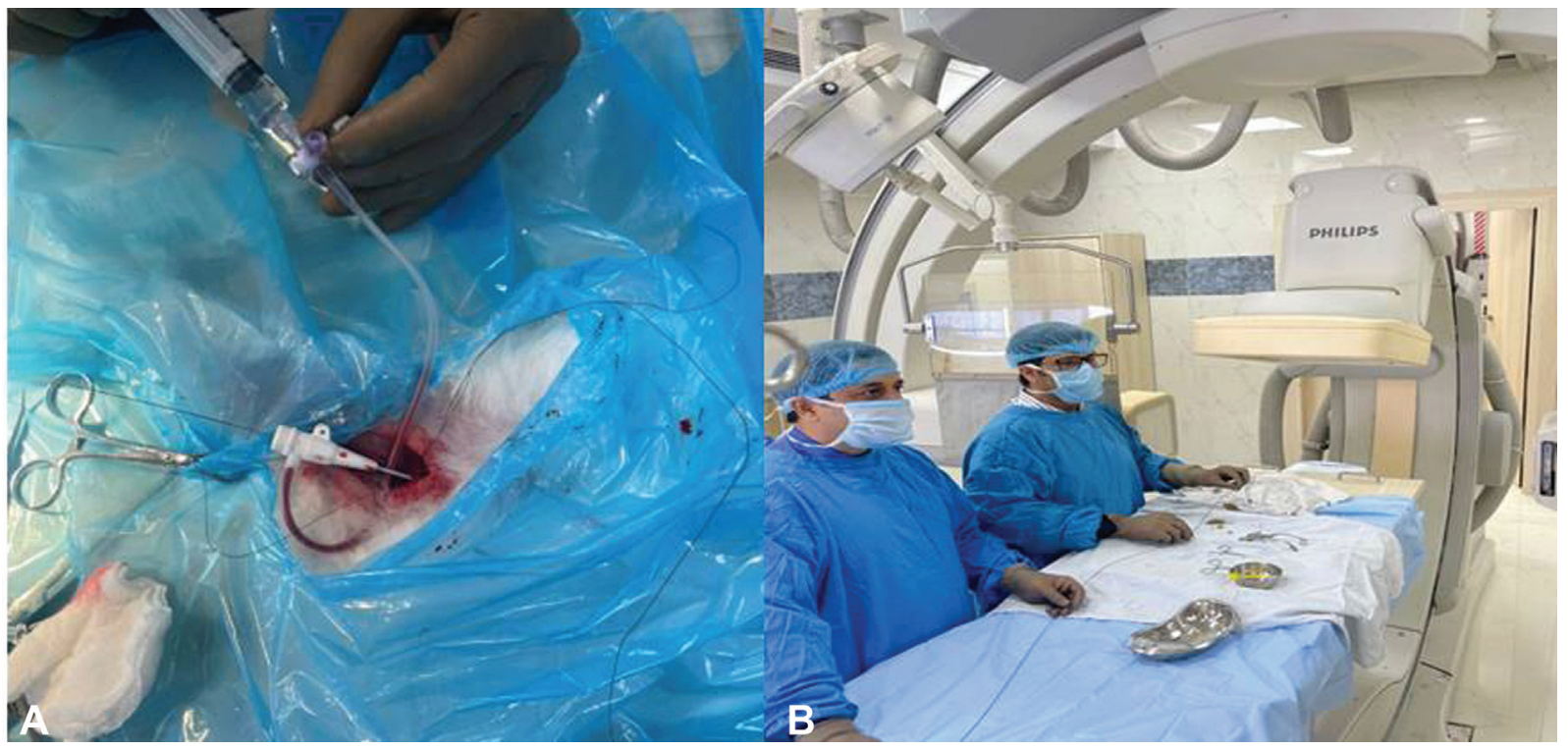

Fig. 3 (A) The presence of a 3F sheath in the right femoral artery after careful dissection. (B) Placement of a 1.7-F microcatheter through the $3 \mathrm{~F}$ sheath for selective angiography.

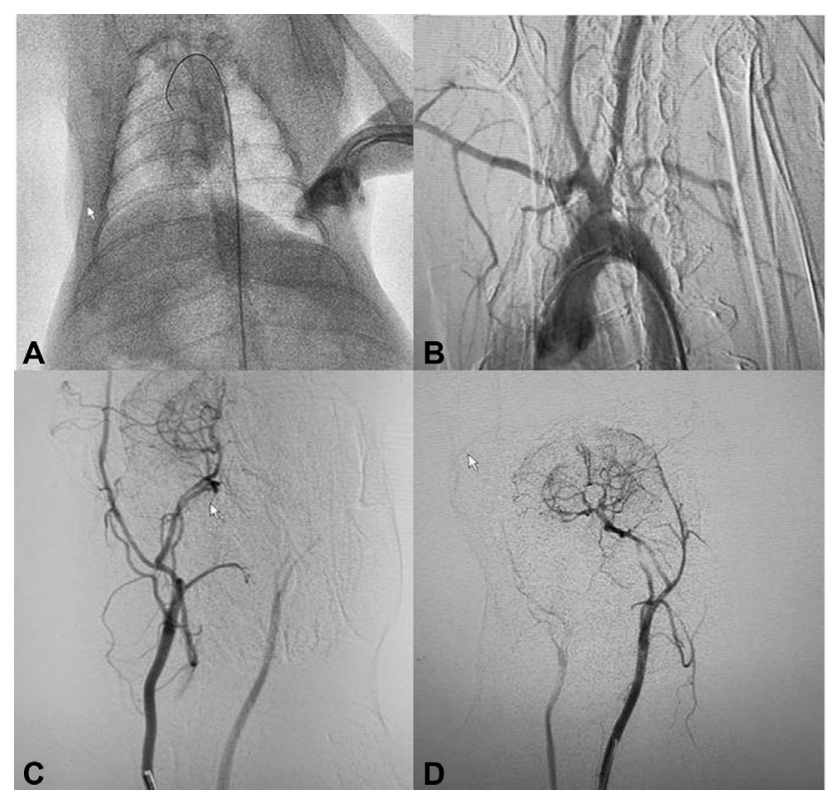

Fig. 4 (A) Fluoroscopic image showing guidewire and microcatheter in the arch of aorta. (B) Aortogram run shows opacification of major branches. Selective common carotid angiogram taken on right (C) and left (D) sides.

\section{Conclusion and Future Direction}

A vast literature is available depicting the clinical utility of pre-clinical studies performed on large animals. ${ }^{4}$ With the establishment of biplane DSA, we hope for increasing our technical expertise in animal vascular procedures. The aim is to provide models for research and development to conduct pre-clinical studies.
Note

We state that this article has not been submitted to any other journal and is our original work.

Ethical Approval

Ethical approval was obtained from Department of Radiodiagnosis and Imaging, Post Graduate Institute of Medical Education \& Research, Chandigarh, India.

Funding

None.

Conflict of Interest

None declared.

\section{References}

1 Altes TA, Cloft HJ, Short JG, et al; American Roentgen Ray Society. 1999 ARRS Executive Council Award. Creation of saccular aneurysms in the rabbit: a model suitable for testing endovascular devices. Am J Roentgenol 2000;174(02): 349-354

2 Culp BC, Brown AT, Erdem E, Lowery J, Culp WC. Selective intracranial magnification angiography of the rabbit: basic techniques and anatomy. J Vasc Interv Radiol 2007;18(02): 187-192

$3 \mathrm{Kim} \mathrm{HJ}$, Choi SH, Kim SH, et al. Cerebral angiography using transauricular access in a rabbit model: a new technique. Acta Radiol 2021;62(01):113-119

4 Herrmann AM, Meckel S, Gounis MJ, et al. Large animals in neurointerventional research: A systematic review on models, techniques and their application in endovascular procedures for stroke, aneurysms and vascular malformations. J Cereb Blood Flow Metab 2019;39(03):375-394 
